\title{
A new consensus-based unemployment indicator
}

\author{
Oscar Claveria ${ }^{1 *}$ \\ ${ }^{1}$ AQR-IREA, University of Barcelona (UB)
}

In this study we present a novel approach to measure the level of consensus among agents' expectations. The proposed framework allows us to design a positional indicator that gives the percentage of agreement between survey expectations. While other aggregation methods such as the balance, which is constructed as the difference between the percentages of respondents giving positive and negative replies, explicitly omit the neutral information, the proposed metric allows synthesizing the information coming from all response categories, including the percentage of respondents who do not expect any change. In order to assess the performance of the proposed measure of consensus, we compare its ability to track the evolution of unemployment to that of the balance in eight European countries. With this aim, we scale both measures to generate oneperiod ahead forecasts of the unemployment rate. We find that the consensus-based unemployment indicator outperforms the balance in all countries except Denmark and Sweden, which suggests that the level of agreement among agents' expectations is a good predictor of unemployment.

JEL Classification: C53; D12; E24; J01; J60

Keywords: unemployment; leading indicator; tendency surveys; consumer expectations; geometry

\section{Acknowledgements}

This research was supported by by the projects ECO2016-75805-R from the Spanish Ministry of Economy and Competitiveness. We would like to thank the Editor and an anonymous referee for their useful comments and suggestions.

\footnotetext{
* Corresponding Author:

Oscar Claveria, University of Barcelona, 08034 Barcelona, Spain. Email: oclaveria@ub.edu
} 


\section{Introduction}

Unemployment is a variable of fundamental interest to economic agents. Since the beginning of the 2008 financial crisis there has been a renewed interest in correctly anticipating the unemployment rate. Expectations have proved useful to assess actual employment changes (Abberger 2007; Claveria, Pons, and Ramos 2007; Lehmann and Weyh 2016). Survey expectations do not suffer from major revisions or measurement errors, and are available about one quarter ahead of the publication of quantitative official data, which makes them particularly suitable for short-term forecasting. Survey results are aggregated in the form of balances, obtained as the difference between the percentages of respondents giving positive and negative replies. The use of the balance is justified by the fact that it is positively related to the first difference of the quantitative variable of reference.

Claveria, Monte, and Torra (2017) and Lahiri and Zhao (2015) link quantified survey expectations to quantitative realizations and obtain a significant improvement in accuracy during periods of uncertainty with high levels of disagreement between respondents. Sakutukwa and Yang (2018) find that uncertainty measures contain useful information to improve short-term forecasts of employment. These results have led us to devise a geometric framework that allows to measure uncertainty as the percentage of disagreement among survey respondents incorporating the information coming from the neutral responses.

Since economic uncertainty is not directly observable, two major strategies have been followed to measure it: tracking the magnitude of forecast errors of macroeconomic variables (Jurado, Ludvigson, and $\mathrm{Ng} 2015$ ) or developing dispersion-based indicators. This second approach can either be based on stock market volatility (Bloom, 2009) or on agents' economic expectations (Mokinski, Sheng, and Yang 2015).

Direct measures of expectations can only be derived from surveys. By using agents' expectations coming from economic tendency surveys, Bachman, Elstner, and Sims (2013) propose several uncertainty indicators based on the dispersion of respondents' expectations about the future in Germany and the United States. For the Euro Area, Girardi and Reuter (2017) design three new dispersion-based disagreement indicators derived from business and consumer surveys. 
These dispersion-based indicators of uncertainty exclusively use the information from the respondents expecting a variable to rise and to fall, leaving out the the responses from agents that do not expect any change. With the aim of overcoming this omission, we design a measure of agreement among agents' expectations that conveys a geometric interpretation. Since Lolić and Sorić (2018) show that the number of response categories plays a pivotal role on the forecast accuracy of quantified consumers' expectations, we use a four-dimensional simplex in the form of a regular pentagon to project five response categories.

The centre of the simplex corresponds to the point of minimum consensus among respondents. Conversely, the fact that the coordinates on the simplex are near a vertex is indicative that there is a high level of agreement about the evolution of the target variable. By linking the distance of each combination of responses for a given period to the distance from the barycentre to the nearest vertex, we obtain the percentage of agreement among respondents.

We use the consensus-based indicator to generate quantitative estimates of the unemployment rate from qualitative survey-based expectations about employment. Finally, we evaluate its performance in eight European countries by comparing it to the balance statistic.

\section{Methodology}

In this section we present a methodology to derive positional indicators of consensus among survey respondents. The framework is based on a recent geometric application to determine the likelihood of disagreement among election outcomes (Saari 2008). See García-Lapresta and Pérez-Román $(2017,2018)$ for different approaches to measure consensus. The proposed approach allows to deal with questions that have five reply options. Hence, vector $X$ contains all the information from the surveyed units at a given time $t$ :

$$
X_{t}=\left(P P_{t}, P_{t}, E_{t}, M_{t}, M M_{t}\right)
$$

Where $P P_{t}$ is the percentage of respondents reporting a sharp increase in the variable, $P_{t}$ a slight increase, $E_{t}$ no change, $M_{t}$ a slight fall, and $M M_{t}$ a sharp fall. The most common way of presenting survey data is the balance, which in the case of five reply options is computed as a weighted mean as follows: 


$$
B_{t}=\left(P P_{t}+1 / 2 P_{t}\right)-\left(1 / 2 M_{t}+M M_{t}\right)
$$

It can be seen that category $E_{t}$ is omitted in the calculation of $B_{t}$. This reduction in the vector of replies implies a loss of the information concerning the degree of certainty of the respondents. In order to overcome this limitation, we develop a methodological framework to construct a measure of consensus that conveys a geometrical interpretation and allows to incorporate the share of neutral responses.

The proposed metric presents two inherent advantages. On the one hand, it allows to capture the trajectories of the five states. On the other hand, it has a self-explanatory interpretation, as it provides the percentage of agreement among respondents. As the sum of the reply options adds to 100 , a natural representation of $X_{t}$ is as a point on a simplex (Coxeter, 1969). In order to explicitly incorporate the five components of $X_{t}$, we use a four-dimensional simplex in the form of a regular pentagon (Fig. 1).

Fig. 1. Simplex - Regular pentagon

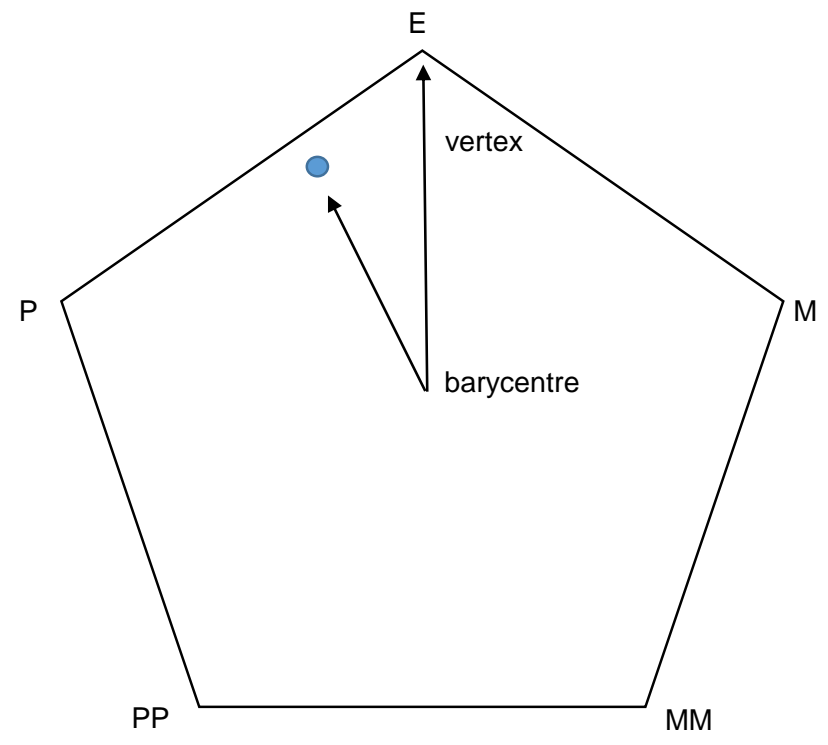

Notes: The five reply options are E (\% of "remains constant" replies), P (\% of "slight increase"), PP (\% of "sharp increase"), MM (\% of "sharp fall"), and M ("slight fall"). The grey point in the simplex corresponds to a unique convex combination of the five reply options for a given period in time.

The interior of this simplex encompasses all possible combinations of reply options, which correspond to the barycentric coordinates of each point in time. The centre of the simplex indicates the point of maximum discrepancy among respondents. If we assume that $E_{t}$ can proxy either one of the other four options, when the coordinate is near the 
barycentre, one would expect little agreement among respondents. The barycentric coordinate system allows us to compute the vertical distance of a point in the simplex to the nearest edge. Hence, we define a metric of consensus as the following ratio:

$$
\text { Agreement }=\frac{\text { Distance of the point to the barycentre }}{\text { Distance from the barycentre to the nearest vertex }}
$$

Given that all vertices are at the same distance to the barycentre, this ratio gives the relative weight of the distance of each point to the barycentre of the pentagon. Therefore, in order to obtain a percentage of consensus through the information contained in (1) we can formalise a measure of consensus at a given period $t$ as:

$$
C_{t}=\frac{\sqrt{\left(P P_{t}-20\right)^{2}+\left(P_{t}-20\right)^{2}+\left(E_{t}-20\right)^{2}+\left(M_{t}-20\right)^{2}+\left(M M_{t}-20\right)^{2}}}{\sqrt{4 / 5}}
$$

The indicator reaches the maximum (100\%) when a response category draws all the responses, and the minimum value of zero when the answers are equidistributed among the five response categories. We illustrate this in Table 1.

Table 1. Comparison of aggregation statistics for different response combinations

\begin{tabular}{ccccc|cc}
\hline$P P_{t}$ & $P_{t}$ & $E_{t}$ & $M_{t}$ & $M_{t}$ & $B_{t}$ & $C_{t}$ \\
\hline 20 & 20 & 20 & 20 & 20 & 0.0 & 0.0 \\
0 & 0 & 100 & 0 & 0 & 0.0 & 100.0 \\
50 & 0 & 0 & 0 & 50 & 0.0 & 61.2 \\
10 & 40 & 0 & 40 & 10 & 0.0 & 41.8 \\
\hline
\end{tabular}

Notes: The five reply options are $E$ (\% of "remains constant" replies), $P$ (\% of "slight increase"), $P P(\%$ of "sharp increase"), $M M$ (\% of "sharp fall"), and $M$ ("slight fall"). $B$ refers to the balance statistic and $C$ to the proposed consensus metric.

\section{Results}

The empirical analysis focuses on consumers' expectations about the future evolution of unemployment in eight European countries. We use monthly data from the joint harmonised EU consumer survey conducted by the European Commission, which can be freely downloaded (https://ec.europa.eu/info/business-economy-euro/indicatorsstatistics/economic-databases/business-and-consumer-surveys_en). The quantitative target variable is the unemployment rate. We use seasonally adjusted rates provided by Eurostat (http://ec.europa.eu/eurostat/web/lfs/data/database). The sample period goes from January 2007 to December 2017. 
Fig. 2. Evolution of unemployment rate vs. Unemployment indicators (2007.01-2017.12)

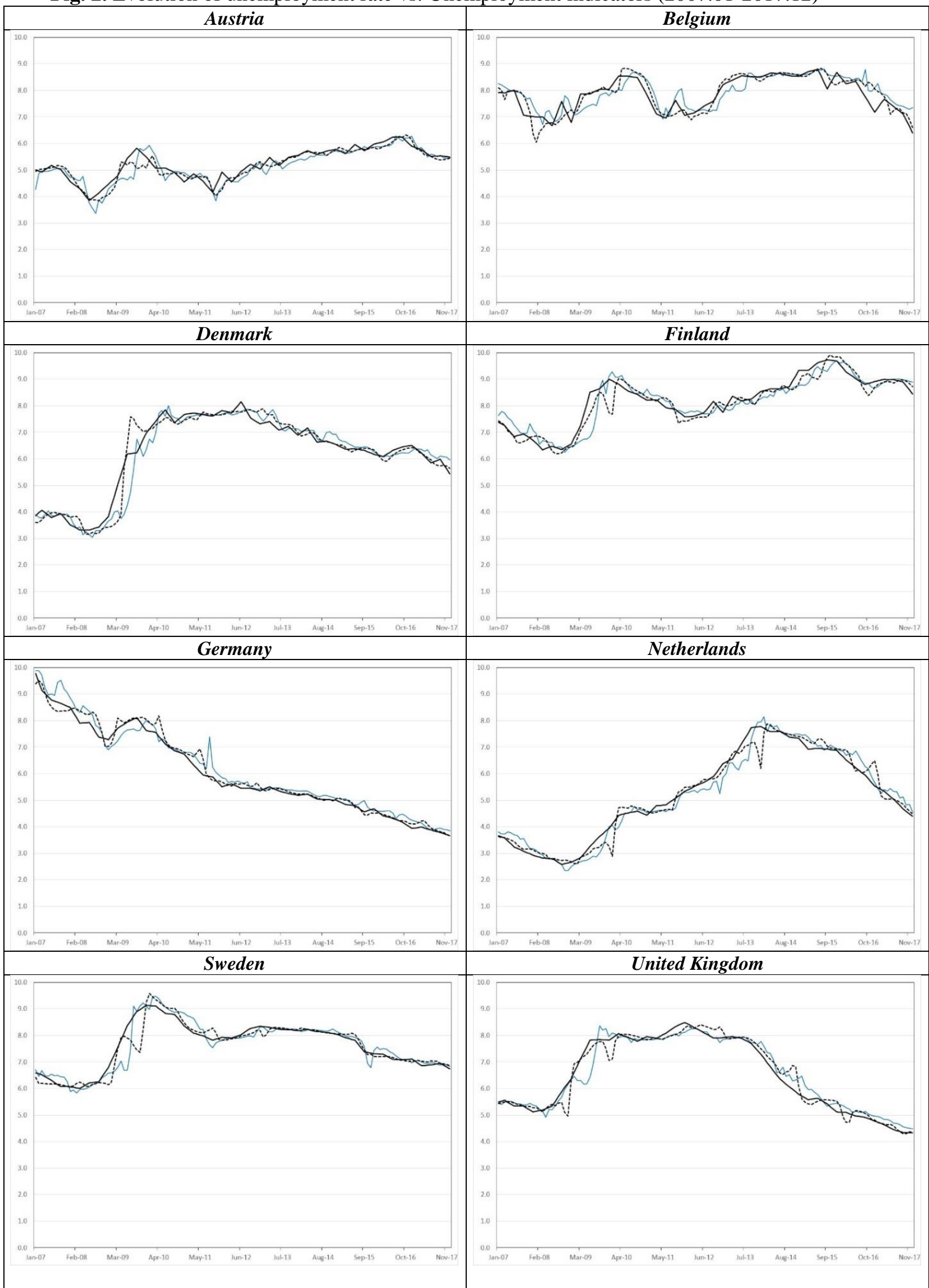

Notes: The black line represents the evolution of the unemployment rate in each country, the blue line the scaled balance (SB), and the black dashed line the consensus-based indicator of unemployment (SC). 
We use the consensus metric to generate estimates of the unemployment rate. With this aim, $C_{t}$ is smoothed with a simple moving average and scaled by means of a rolling regression. As official quantitative data are published with a delay of more than one period with respect to survey data, these estimates can be regarded as one-month ahead out-of-sample forecasts.

In Fig. 2 we compare the evolution of the proposed consensus-based indicator of unemployment $\left(S C_{t}\right)$ to that of the scaled balance $\left(S B_{t}\right)$ and the unemployment rate. We can observe that both indicators show a similar pattern, closely correlated to the oscillations of the unemployment rate.

To evaluate the forecasting performance of both survey-based measures of unemployment expectations, we compute the mean absolute percentage error (MAPE), which scales the absolute error by the actual value of the variable for every point in time. As we are dealing with positive data, and comparing countries with different unemployment rates, this scale-independent measure of accuracy is particularly appropriate (Hyndman and Koehler 2006). Results of this out-of-sample forecasting comparison are presented in Table 2.

Table 2. Forecast accuracy by country (2007.01-2017.12)

\begin{tabular}{lcclll}
\hline & MAPE & \multicolumn{3}{c}{ MAPE } \\
\hline Austria & $S B_{t}$ & $S C_{t}$ & & $S B_{t}$ & $S C_{t}$ \\
Belgium & 4.08 & 3.11 & Germany & 3.82 & 2.58 \\
Denmark & 3.91 & 2.81 & Netherlands & 5.38 & 4.36 \\
Finland & 4.18 & 4.91 & Sweden & 2.65 & 2.95 \\
\hline
\end{tabular}

Notes: MAPE stands for the mean percentage absolute error. SB refers to the scaled balance statistic, and $\mathrm{SC}$ to the proposed leading indicator of unemployment based on a measure of consensus among consumer expectations.

The results in Table 2 show that the proposed unemployment indicator outperforms the scaled balance in all countries except Denmark and Sweden. The lowest MAPE values are obtained in Belgium, Germany, Finland and Sweden. These results are in line with those of Lehmann and Wohlrabe (2017), who find that consumers' unemployment expectations exhibit a high forecasting accuracy in Germany. For this same country, Hutter and Weber (2015) show that exploiting the serial correlation in the response behaviour of experts' unemployment expectations yields better forecasting results. Martinsen, Ravazzolo, and Wulfsberg (2014) and Österholm (2010) also find evidence 
that unemployment expectations help to improve unemployment forecasting in Norway, Sweden and Finland.

\section{Conclusion}

This paper presents a geometric approach to derive a novel measure of consensus among agents' survey-based expectations. We scale this metric to generate estimates of the unemployment rate from qualitative consumer expectations in eight European countries. With the aim of assessing its performance, we compare its ability to anticipate unemployment to that of the balance. Given that the proposed indicator leads to an improvement in the accuracy of forecasts in all countries except Denmark and Sweden, it seems that the level of consensus among respondents contains useful information to forecast unemployment.

\section{References}

Abberger, K. 2007. "Qualitative business surveys and the assessment of employment - A case study for Germany." International Journal of Forecasting 23: 249-258.

Bachmann, R., Elstner, S., and Sims, E. R. 2013. "Uncertainty and economic activity: Evidence from business survey data." American Economic Journal: Macroeconomics 5: 217-249.

Bloom, N. 2009. "The impact of uncertainty shocks." Econometrica 77: 623-685.

Claveria, O., Monte, E., and Torra, S. 2017. "Assessment of the effect of the financial crisis on agents' expectations through symbolic regression." Applied Economics Letters 24: 648652.

Claveria, O., Pons, E., and Ramos, R. 2007. "Business and consumer expectations and macroeconomic forecasts." International Journal of Forecasting 23: 47-69.

Coxeter, H. S. M. 1969. Introduction to Geometry (2nd Edition). London: John Wiley \& Sons.

García-Lapresta, J. L., and Pérez-Román, D. 2017. "Consensus-based agglomerative hierarchical clustering." In: Fuzzy Sets, Rough Sets, Multisets and Clustering., edited by V. Torra, A. Dahlbom, and Y. Narukawa, 123-135. Studies in Computational Intelligence 671. Cham: Springer.

García-Lapresta, J. L., and Pérez-Román, D. 2018. "Aggregating opinions in non-uniform ordered qualitative scales." Applied Soft Computing 67: 652-657.

Girardi, A., and Reuter, A. 2017. "New uncertainty measures for the euro area using survey data." Oxford Economic Papers 69: 278-300.

Hutter, C., and Weber, E. 2015. "Constructing a new leading indicator for unemployment from a survey among German employment agencies." Applied Economics 47: 3540-3558.

Hyndman, R.J., and Koehler, A.B. 2006. "Another look at measures of forecast accuracy." International Journal of Forecasting 22: 679-688.

Jurado, K., Ludvigson, S., and Ng, S. 2015. "Measuring uncertainty.” American Economic Review 105: 1177-216. 
Lahiri, K., and Zhao, Y. 2015. "Quantifying survey expectations: A critical review and generalization of the Carlson-Parkin method." International Journal of Forecasting 31: 5162.

Lehmann, R., and Weyh, A. 2016. "Forecasting employment in Europe: Are survey results helpful?" Journal of Business Cycle Research 12: 81-117.

Lehmann, R., and Wohlrabe, K. 2017. "Experts, firms, consumers or even hard data? Forecasting employment in Germany." Applied Economics Letters 24: 279-283.

Lolić, I., and Sorić, P. 2018. "A critical re-examination of the Carlson-Parkin method." Applied Economics Letters. In Press.

Martinsen, K., Ravazzolo, F., and Wulfsberg, F. 2014. "Forecasting macroeconomic variables using disaggregate survey data." International Journal of Forecasting 30: 65-77.

Mokinski, F., Sheng, X., and Yang, J. 2015. "Measuring disagreement in qualitative expectations." Journal of Forecasting 34: 405-426.

Österholm, P. 2010. "Improving unemployment rate forecasts using survey data." Finnish Economic Papers 23: 16-26.

Saari, D. G. 2008. "Complexity and the geometry of voting." Mathematical and Computer Modelling 48: 551-573.

Sakutukwa, T., and Yang, H-S. 2018. "The role of uncertainty in forecasting employment by skill and industry." Applied Economics Letters. In Press. 